\title{
ESTIMATIVA DA EVAPOTRANSPIRAÇÃO DE REFERÊNCIA E DA DEMANDA SUPLEMENTAR DE IRRIGAÇÃO PARA O MILHO (Zea mays L.) EM SEROPÉDICA E CAMPOS, ESTADO DO RIO DE JANEIRO
}

\author{
Marcos Alexandre Amaral de Oliveira ${ }^{1}$ e Daniel Fonseca de Carvalho ${ }^{2}$
}

\begin{abstract}
RESUMO
Realizou-se um estudo com o objetivo de se avaliar a correlação entre diferentes métodos utilizados na estimativa da evapotranspiração de referência (ETo) e seu efeito na demanda de irrigação suplementar para a cultura do milho, nos municípios de Seropédica e Campos, RJ. Foram ajustadas equações de regressão linear, tendo como variável dependente, a ETo obtida pelo método de Penman-Monteith, considerado padrão e, como variável independente, a ETo obtida pelos outros métodos estudados, obtendo-se coeficientes de correlação iguais ou maiores que $95 \%$. Os resultados mostraram um bom ajuste nos métodos de FAO-Penman, FAO-Radiação e Hargreaves, comparando-os com o método de Penman-Monteith; além do mais, o ajuste da equação de Penman-Monteith com os dados obtidos da equação de Hargreaves forneceu estimativas satisfatórias da demanda de irrigação suplementar para diferentes épocas de plantio, nas duas localidades estudadas.
\end{abstract}

Palavras-chave: evapotranspiração, Penman-Monteith, irrigação suplementar

\author{
ESTIMATIVE OF REFERENCE EVAPOTRANSPIRATION AND OF \\ SUPPLEMENTAL IRRIGATION DEMAND FOR CORN (Zea mays L.), IN \\ SEROPÉDICA AND CAMPOS, RIO DE JANEIRO STATE
}

\begin{abstract}
A study was carried out to evaluate different methods used for estimation of reference evapotranspiration (ETo) and its effect on supplemental irrigation depth for corn, in the municipal districts of Seropédica and Campos, Rio de Janeiro, Brazil. Equations of linear regression were fitted having as dependent variable the Eto values obtained by the Penman-Monteith (PM) method, considered standard, and as the independent variable the ETo values obtained by the other studied methods. The results showed a good fitting to FAO-Penman, FAO-Radiation and Hargreaves (HG) methods, when compared to the Penman-Monteith (PM) method. Also, the fitting of the PM equation from HG data gave a good estimation of the demand of supplemental irrigation for different growing seasons in the two studied places.
\end{abstract}

Key words: evapotranspiration, Penman-Monteith, supplemental irrigation

\section{INTRODUÇÃO}

O Estado do Rio de Janeiro apresenta grande potencial agrícola, porém as precipitações pluviométricas, como em outras regiões do país, não atendem às necessidades hídricas das plantas cultivadas na maior parte do ano. De acordo com Bernardo (1995) o uso da irrigação suplementar se constitui numa importante alternativa para o desenvolvimento da agricultura

\footnotetext{
${ }^{1}$ Estudante de Agronomia, Universidade Federal Rural do Rio de Janeiro. E-mail: marcos alexandre@infolink.com.br

2 Prof. Adjunto, Departamento de Engenharia/Instituto de Tecnologia/UFRRJ. E-mail: carvalho@ufrrj.br
} 
nessas regiões, diminuindo assim os riscos de perdas de produção.

Em projetos de irrigação, o manejo adequado da água é de grande importância, pois a sua captação e elevação exigem alto investimento em equipamentos e grande consumo de energia, além da possibilidade de gerar impactos ambientais nas regiões de intensa exploração da agricultura irrigada e por esses motivos, a quantificação da água evapotranspirada num sistema solo-planta-atmosfera, torna-se numa informação primordial nos projetos de irrigação, determinando o sucesso do empreendimento, tanto economicamente quanto em termos ambientais. De posse desta informação, pode-se determinar corretamente a quantidade e o momento da aplicação de água pela irrigação, maximizando o uso dos recursos hídricos de uma região.

A estimativa da evapotranspiração de referência (ETo) de uma localidade constitui-se numa dificuldade ao planejamento correto da irrigação, pois para a maior parte dos métodos utilizados são necessários dados climatológicos e/ou coeficientes de ajuste normalmente não disponíveis ao irrigante; além disso, existem problemas de sub ou superestimativas da lâmina evapotranspirada.

Pesquisas conduzidas em diferentes localidades e condições climáticas indicam que o método de Penman-Monteith tem apresentado estimativas de ETo para a grama, bem correlacionadas com valores obtidos em lisímetros; portanto, este método pode ser utilizado satisfatoriamente na determinação da ETo sendo recomendado para calibrar outros métodos (Alves Sobrinho et al., 1996).

Tendo em vista a necessidade de um grande número de variáveis climáticas na estimativa da ETo por PenmanMonteith, desenvolveu-se este trabalho com o objetivo de avaliar a correlação entre diferentes métodos utilizados na determinação da ETo e seu efeito na demanda de irrigação suplementar para o milho, nos municípios de Seropédica e Campos (RJ).

\section{MATERIAL E MÉTODOS}

No presente trabalho foram considerados dois municípios no Estado do Rio de Janeiro: Seropédica, localizada a 22 $48^{\circ}$ de latitude sul e $43^{\circ} 41^{\prime}$ de longitude oeste, e Campos, localizada a $21^{\circ} 45^{\prime}$ ' de latitude sul e $41^{\circ} 20^{\prime}$ de longitude oeste. No primeiro, encontra-se o campus da Universidade Federal Rural do Rio de Janeiro (UFRRJ) e em Campos está localizada uma Estação Experimental da UFRRJ, considerada importante região agrícola do Estado. Nos dois municípios encontram-se estações climatológicas do INMET, cadastradas sob os números 83741 e 83698 , respectivamente, de onde foram retirados dados diários observados no período de 1961 a 1978 .

A evapotranspiração de referência (ETo) foi estimada diariamente pelos métodos de Penman-Monteith (PM), padrão FAO, considerado padrão (Smith, 1991), Penman-FAO (PN), Radiação-FAO (RA) e Hargreaves (HG) (Jensen et al., 1990) utilizando um software desenvolvido por Carvalho (1998).
Com base nos valores diários de ETo, foram ajustadas equações de regressão linear tendo-se como variáveis dependentes, os valores de ETo (PM) e, como variável independente, os valores de ETo estimados pelos outros métodos. A partir desta análise, foram determinados o erropadrão de estimativa do método (EES) e o erro-padrão de estimativa ajustado pela regressão (AEES) conforme Jensen et al. (1990).

Utilizando-se o mesmo software (Carvalho, 1998) estimou-se a demanda de irrigação suplementar para a cultura do milho para diferentes épocas de plantio, nas duas localidades, a fim de se verificar a influência do método de cálculo da evapotranspiração (PM, HG e PM, ajustada por meio de HG) na lâmina de irrigação. Para cada dia do ano, o plantio da cultura do milho foi simulado e a duração do ciclo foi estimada utilizando-se o conceito de graus/dia, descrito por Costa (1991).

\section{RESULTADOS E DISCUSSÃO}

Na Figura 1 é apresentada a comparação entre as estimativas de ETo pelos métodos Hargreaves, Radiação e Penman, em relação ao método de Penman-Monteith, para as duas localidades estudadas.

Apesar das diferenças climáticas existentes entre os dois municípios, as estimativas de ETo por HG, RA e PN, em relação à $\mathrm{PM}$, tiveram comportamento semelhante, superestimando a estimativa padrão.

Os coeficientes das equações de regressão ajustadas, os coeficientes de correlação, os valores de EES e AEES e a variação percentual das estimativas médias em relação ao método-padrão, são apresentados na Tabela 1 .

O método da Radiação-FAO, foi aquele que apresentou o menor EES para as duas localidades. Quanto ao ajuste pela equação de regressão linear, todos os métodos apresentaram boa correlação, com valores de $r^{2}$ iguais ou maiores que $95 \%$. Este mesmo resultado foi observado por Alves Sobrinho et al. (1996) e Dantas Neto (1997) para localidades nos Estados de Mato Grosso do Sul e Rio Grande do Norte, respectivamente.

As Figuras 2 e 3 apresentam a variação da ETo estimada pelos métodos estudados ao longo do ano, para Seropédica e Campos, respectivamente. Pode-se verificar que os métodos HG, RA e PN superestimam o método PM (padrão) ao longo de todo o ano, para as duas localidades; já os HG e RA proporcionaram estimativas de ETo semelhantes nos meses de abril a agosto, período em que o método de $\mathrm{PN}$ apresentou estimativas semelhantes ao HG e RA somente para Campos (Figura 3). Nas duas localidades o método de PN apresentou maior variação ao longo do ano.

Nas Figuras 4 e 5 são apresentadas as demandas médias mensais de irrigação suplementar para cultura do milho, nos municípios de Seropédica e Campos, respectivamente. A lâmina de irrigação foi estimada considerando-se os métodos de HG, PM e a estimativa de PM, pela equação de regressão ajustada de $\mathrm{HG}$, em razão da necessidade de dados de temperaturas máxima e mínima para o método HG. 

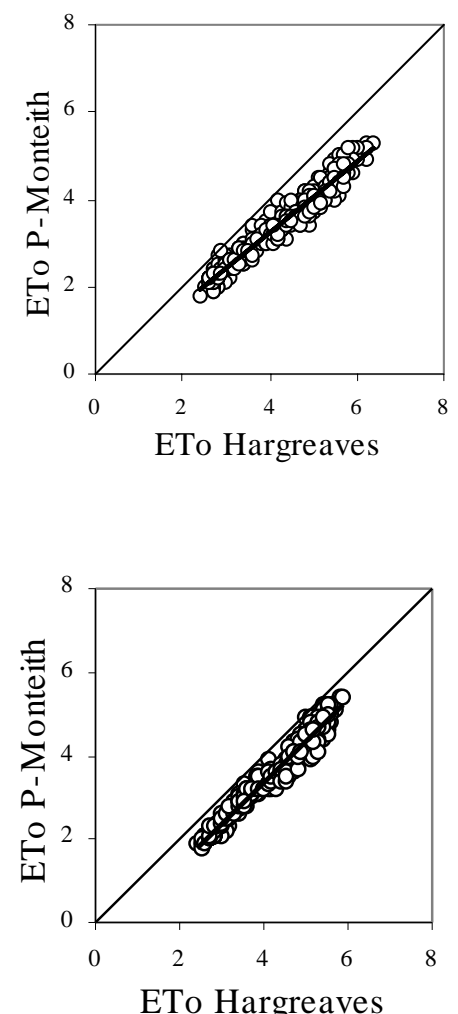

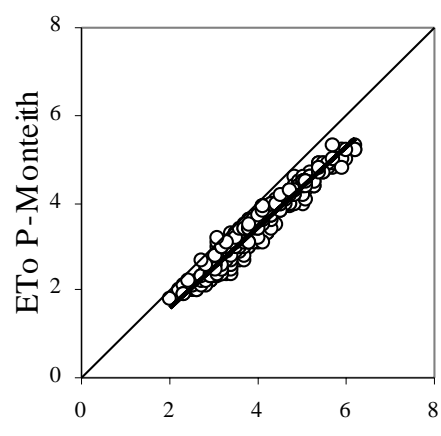

ETo Radiação-FAO

a. Seropédica

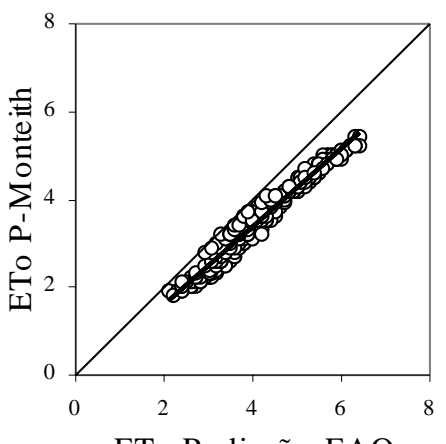

ETo Radiação-FAO

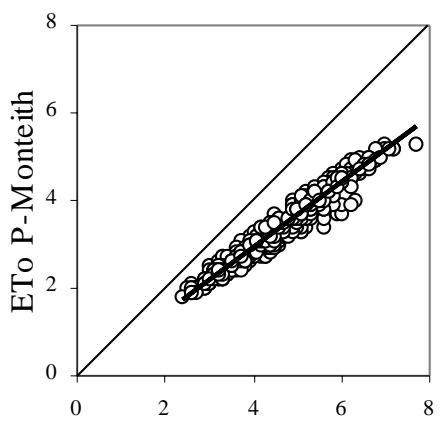

ETo Penman-FAO

$$
\text { b. Campos }
$$

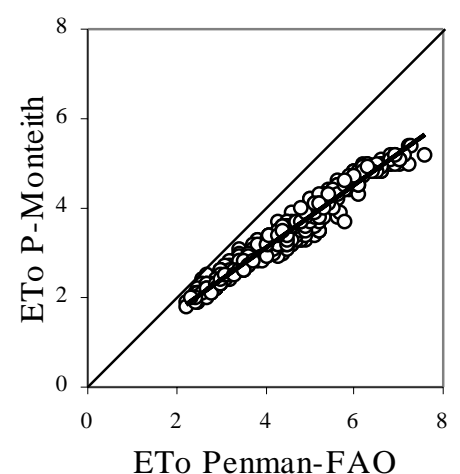

Figura 1. Comparação entre os valores diários de ETo estimados para Seropédica e Campos, para os anos de 1961 a 1978

Tabela 1. Resumo das análises estatísticas entre os métodos de estimativa da evapotranspiração de referência estudados

\begin{tabular}{|c|c|c|c|c|c|c|}
\hline \multirow[t]{2}{*}{ Local/Método } & \multirow[t]{2}{*}{$\%^{(1)}$} & $\mathrm{EES}^{2}$ & $a^{3}$ & $b^{4}$ & $\mathrm{r}^{2} 5$ & \multirow[t]{2}{*}{ AEES $^{6}$} \\
\hline & & \multicolumn{4}{|c|}{ ETo $(\mathrm{PM})=\mathrm{a}+\mathrm{b} . \mathrm{ETo}($ método $)$} & \\
\hline \multicolumn{7}{|l|}{ SEROPÉDICA } \\
\hline Penman - FAO & 136 & 1,30 & $-0,0466$ & 0,7441 & 0,95 & 0,20 \\
\hline Hargreaves & 123 & 0,85 & $-0,0230$ & 0,8161 & 0,95 & 0,19 \\
\hline Radiação - FAO & 116 & 0,59 & $-0,2234$ & 0,9176 & 0,95 & 0,19 \\
\hline \multicolumn{7}{|l|}{ CAMPOS } \\
\hline Penman - FAO & 130 & 1,12 & 0,3035 & 0,7048 & 0,96 & 0,19 \\
\hline Hargreaves & 118 & 0,66 & $-0,5568$ & 0,9827 & 0,96 & 0,18 \\
\hline Radiação - FAO & 118 & 0,66 & $-0,2056$ & 0,8970 & 0,97 & 0,17 \\
\hline
\end{tabular}

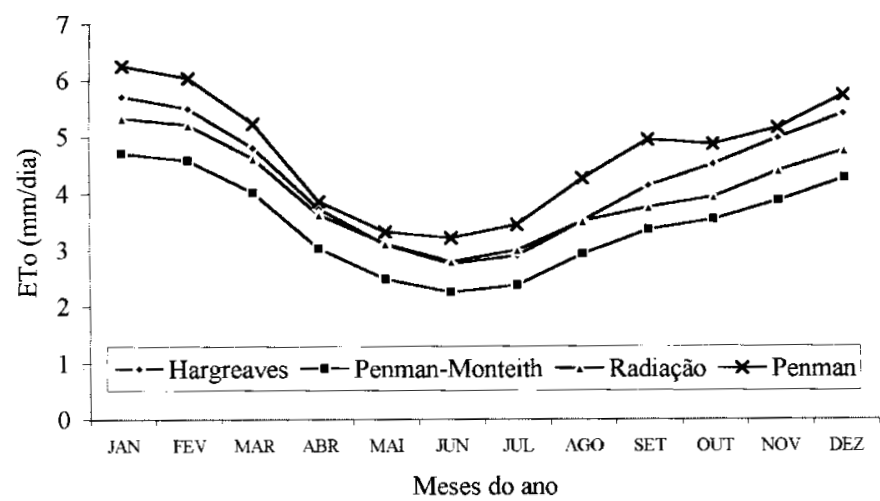

Figura 2. Evolução dos valores médios mensais de ETo estimados pelos métodos estudados para Seropédica

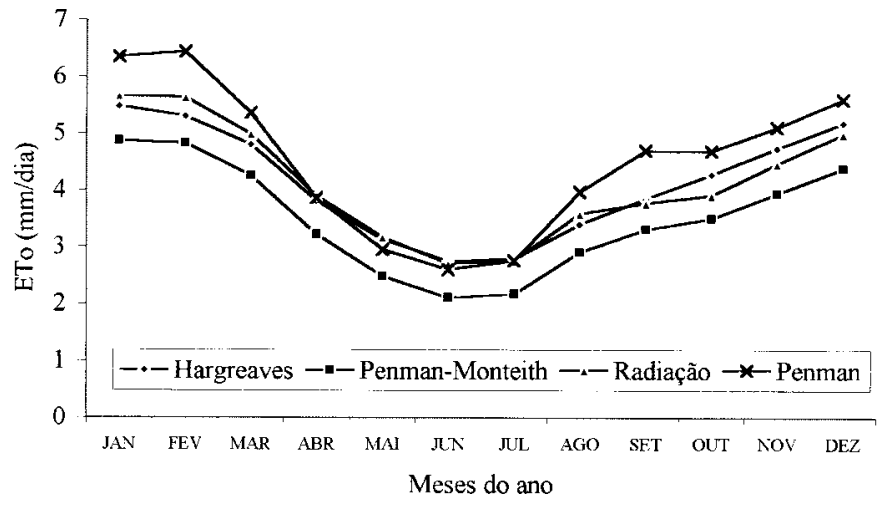

Figura 3. Evolução dos valores médios mensais de ETo estimados pelos métodos estudados para Campos 


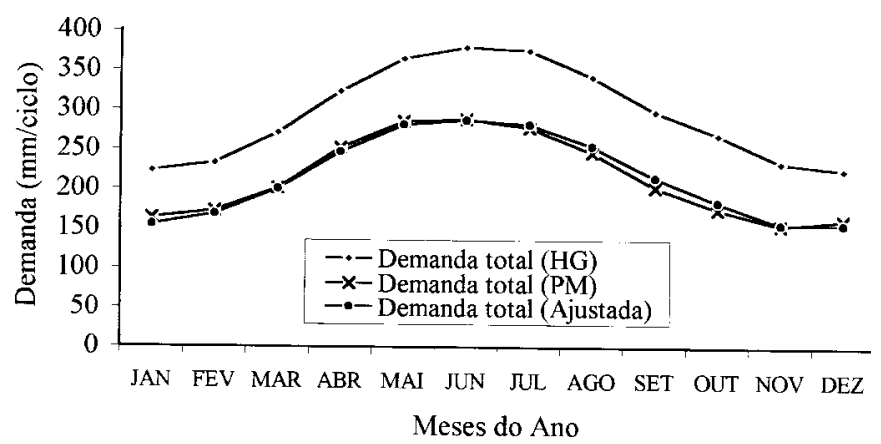

Figura 4. Demanda de irrigação suplementar para a cultura do milho considerando-se diferentes épocas de plantio, para diferentes métodos de estimativa de ETo, para Seropédica

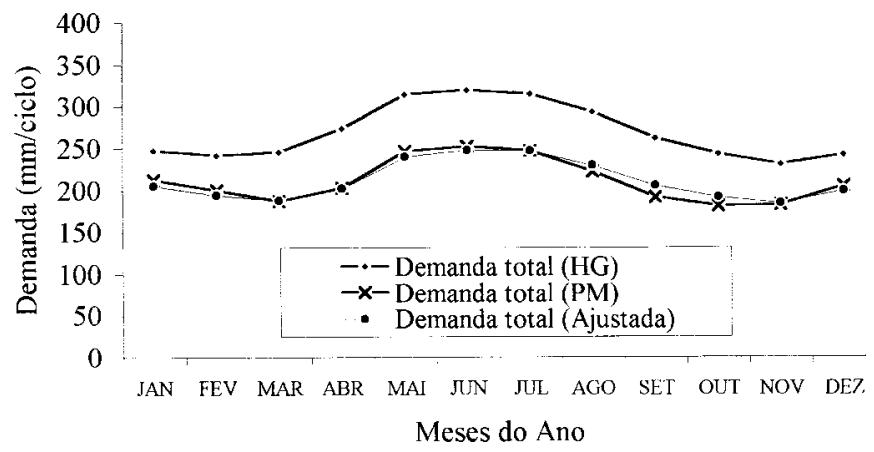

Figura 5. Demanda de irrigação suplementar para a cultura do milho considerando-se diferentes épocas de plantio, para diferentes métodos de estimativa de ETo, para Campos

A demanda de irrigação suplementar estimada, considerando-se a ETo calculada por HG apresentou, em média, valores 38,2 e $27,8 \%$ superiores àqueles obtidos com a ETo calculada por PM, para Seropédica e Campos, respectivamente. A diferença máxima observada foi de 53,8 \% para Seropédica, em outubro, e de $36,8 \%$ para Campos, no mês de setembro.

Considerando-se a equação ajustada de PM com os dados de HG na estimativa da demanda suplementar de irrigação, pode-se verificar valores semelhantes àqueles obtidos com 0 método de PM, para as duas localidades. As diferenças observadas foram, em média, de 0,13\% em Seropédica e de $0,32 \%$ em Campos. A diferença máxima observada para Seropédica foi de $5,81 \%$ e Campos de $7,1 \%$, ambas no mês de setembro, comprovando a boa correlação entre as ETo estimadas por PM e HG.

Em razão de maiores médias de temperatura, a duração do ciclo para a cultura do milho foi sensivelmente inferior no município de Campos em comparação com a de Seropédica. Este fato pode ter favorecido menor demanda suplementar de irrigação para aquele município, uma vez que as lâminas de precipitação dependente para as duas localidades apresentaramse semelhantes.

\section{CONCLUSÕES}

Com base nos resultados obtidos, pode-se concluir que:

1. Os métodos de estimativa de ETo estudados superestimaram o método-padrão (PM) nas duas localidades.

2. Em relação ao método padrão, todos os demais apresentaram boa correlação, com valores de $\mathrm{r}^{2}$ iguais ou maiores que $95 \%$, permitindo a utilização das equações como forma de estimativa de ETo, nas duas localidades estudadas.

3. A utilização da equação ajustada de PM com os dados de HG permitiu estimativas satisfatórias de demanda de irrigação suplementar para diferentes épocas de plantio, em Seropédica e em Campos.

\section{REFERÊNCIAS BIBLIOGRÁFICAS}

ALVES SOBRINHO, T.; BONOMO, R; MANTOVANI, E.C.; SEDIYAMA, G. C. Estimativa da evapotranspiração de referência (ETo) para Dourados e Ponta Porã, Mato Grosso do Sul. In: CONGRESSO BRASILEIRO DE ENGENHARIA AGRÍCOLA, 25, 1996, Bauru. Resumos... Bauru: Sociedade Brasileira de Engenharia Agrícola, 1996. CD Rom.

BERNARDO, S. Manual de irrigação. 6⿳a ed. Viçosa: Imprensa Universitária, 1995. 657p.

CARVALHO, D.F. Otimização do uso da água no perímetro irrigado do Gorutuba. Viçosa, MG: UFV, 1998. Tese Doutorado (no prelo).

COSTA, M. H. Modelo de otimização dos recursos hídricos para a irrigação, conforme a época de plantio. Viçosa, MG: UFV, 1991. 111p. Dissertação Mestrado.

DANTAS NETO, F.S. Avaliação de métodos para estimativa da evapotranspiração de referência para Mossoró, RN. In: CONGRESSO BRASILEIRO DE ENGENHARIA AGRÍCOLA, 26, 1997, Campina Grande. Resumos... Campina Grande: Sociedade Brasileira de Engenharia Agrícola, 1997. CD Rom.

JENSEN, M.E.; BURMAN, R.D.; ALLEN, R.G. Evapotranspiration and irrigation water requeriments. New York: ASCE, 1990. 332p.

SMITH, M. Report on the expert consultation on revision of FAO methodologies for crop water requeriments. Rome: FAO, 1991. 45p. 